\title{
The Role of Mesenchymal Stem Cells Controlling Liver Function in Liver Fibrosis
}

\author{
Arini Dewi Antari ${ }^{1, *}$, Ardi Prasetio ${ }^{2}$ and Baskoro Adi Nugroho ${ }^{3}$
}

\begin{abstract}
Background: Liver fibrosis (LF) is the end-stage of liver damage characterized by the increase concentration of SGOT and SGPT. A previous study, revealed that liver transplantation as the most effective therapy for LF has limited availability and remains a major challenge. Mesenchymal stem may control inflammation and regenerate damaged tissue leading to accelerated liver function repair.

Aim: The purpose of this study was to determine the optimum doze for MSC administration to recover LF. Method: The research used variations doze of MSCs administration of $1 \times 10^{6}$ and $2 \times 10^{6}$. Liver firbosis was induced by twice a week intraperitoneal injection of carbon tetrachloride (CCl4) (Sigma-Aldrich) dissolved with olive oil in 1:1 ratio for eight weeks. The concentration of SGPT SGOT was measured at day 9 post MSC administration. One Way Anova was performed to analyze statictical data.

Result: This study showed that there was a significant decrease in SGOT concentration $(P<0,05)$. P2 group treatment showed the lowest concentration of SGOT among the treatment (Figure B). In the same time, the administration of UC-MSCs reduce SGPT concentration insignificantly $(P>0,05)$.

Conclusion: The transplantation of MSCs ameliorated LF by reducing SGPT and SGOT concentration
\end{abstract}

Keywords: Mesenchymal stem cells, liver fibrosis, SGOT, SGPT

\section{BACKGROUD}

Liver fibrosis (LF) is the end-stage of liver damage, regarding to the excessive accumulation of extracellular matrix (ECM) leading to hepatocellular dysfunction caused by prolonged inflammation ${ }^{1}$. Liver fibrosis is characterized by the increase concentration of Serum Glutamic Oxaloacetic Transaminase (SGOT) and Serum Glutamic Pyruvic Transaminase (SGPT) ${ }^{2}$. Epidemiological data has reported that LF cause over one million death per year or equal to $2 \%$ of total mortality worldwide ${ }^{3}$. A previous study, revealed that Liver transplantation is the most effective therapy for LF disease ${ }^{4}$. Unfortunately, the limited availability of donor livers remains a major challenge. Thus, investigating the alternative treatment to control LF is urgently needed. The cell-based treatment including the administration of mesenchymal stem cells (MSCs) become a major concern since MSCs have a high potency to control inflammation and regenerate damaged tissue leading to accelerated wound healing ${ }^{5-7}$.

Based on International Society of Cellular Therapy, MSCs positively express several specific surface markers such as CD105, CD90, CD73, and negatively exspress CD11b, CD14, CD19 or CD79a, CD45, CD34, as well as Human Leucocyte Antigen (HLA) class II markers ${ }^{8}$. The uumbilical cord derived-MSCs (UCMSCs) possess more a robust stemness gene expression than the other MSCs sources ${ }^{9}$.

\footnotetext{
*Correspondence:

${ }^{1}$ Department of Postgraduate Biomedical Science, Medical Faculty, Sultan Agung Islamic University, Semarang, Indonesia

Full list of author information is available at the end of the article
} 
Several studies have demonstrated that the administration of MSCs can be a key player in liver directed regenerative medicine ${ }^{10-12}$. MSCs become a supportive function needed for regenerative medicine application since it is effective therapy for several health disorders including hepatic disease ${ }^{13,14}$. MSC play crucial role to reduce fibrosis through immunomodulatory properties and paracrine mechanism ${ }^{15}$. Moreover UC-MSCs are able to replace damaged hepatocyte through differentiation capacity and induce the regeneration of damaged cells. Previous study reported that the reduce of SGPT and SGOT showed the recovery of liver function ${ }^{16}$. However the optimum doze for MSC to recover LF is not clear. Therefore, this study aims to determine the optimum dose of MSCs administration to controlling SGOT and SGPT levels associated with liver function improvement on LF mice model.

\section{METHODS}

\section{Fibrosis liver animal model}

All procedures applied in this study were approved by the Ethics Committee of Medical Faculty. Wistarstrain rats $(300 \pm 30 \mathrm{~g}, \mathrm{CV} 10 \%)$ were fed ad libitum, reared at temperature-conditioned room at $24 \square \mathrm{C}$ and $12 \mathrm{~h}$ light-cycle. Liver fibrosis was induced by twice a week intraperitoneal injection of carbon tetrachloride (CC14) (Sigma-Aldrich) dissolved with olive oil in 1:1 ratio for eight weeks. Masson's trichrome staining was performed to confirm the liver fibrosis formation.

\section{Mesenchymal Stem Cell Isolation}

All the isolation protocols were performed under sterile procedure. Umbilical cords were obtained Wistar-strain rat on two-week gestation. The umbilical cord was separated from the blood vessel, minced into 2-3 $\mathrm{mm}$ piece and plated into $25 \mathrm{~cm} 2$ culture flask. The explants were soaked with Dulbecco's Modified Eagle's Medium (DMEM) mixed with 10\% Fetal Bovine Serum (FBS) (Sigma-Aldrich, Louis St, MO), 100 $\mathrm{IU} / \mathrm{mL}$ penicillin, and $100 \mathrm{mg} / \mathrm{mL}$ of streptomycin (GibcoTM Invitrogen, NY, USA) and incubated under $37 \square \mathrm{C}, 5 \% \mathrm{CO} 2$, and $80 \%$ humidity condition. The culture medium was replaced in 3 days interval. After cells reached $80 \%$ confluency, the isolated cells were passaged using trypsin for following MSCs characteristic validation and treatment.

\section{Mesenchymal Stem Cell Characteristic Validation}

The immunophenotype characteristic of the UC-MSC at passage 4 were validated using Flow cytometer Accuri C6 plus (BD science ${ }^{\circledR}$ ). Briefly, the isolated cell was labeled using positive marker CD90 FITC, CD105 PerCP-Cy5.5, CD73 APC, and hematopoietic lineage marker CD45 PE, CD19 PE, CD11b PE, CD34 PE (BD Stemflow $\left.{ }^{\mathrm{TM}}\right)$.

The capability of UC-MSC to differentiate into mature cell, including osteoblast and adipocyte, was analyzed by incubating in Osteogenic and Adipogenicity medium (Stem cell mesencult) for 15 days with threeday interval medium replacement. The calcium deposition was displayed as bright red color among the cell after Alizarin red staining while the lipid formation was shown as red droplets after Oil Red $\mathrm{O}$ staining.

\section{Administration of UC-MSCs}

Liver fibrosis-induced rats were randomly assigned into four treatment group $(n=6)$ and injected by $1 \mathrm{x}$ 106 MSCs (P1), 2 x 106 MSCs (P2), and without treatment (Sham), all these administrations were injected via lateral tail vein.

\section{Liver function test}

Serum Glutamic Oxaloacetic Transaminase (SGOT) and Serum Glutamic Pyruvic Transaminase (SGPT) concentration was determined by commercially available rat enzyme kits to measure liver function condition using Photometry procedure.

\section{Statistical analysis}

One way Analysis of Varian (Anova) was carried out to determine statistical significance and P value $\mathbf{4 3} \mid \mathrm{P}$ a g e 
of less than 0.05 was considered as statistically significant.

\section{RESULTS}

The UC-MSC were plastic adherent and morphologically displayed spindle-like shape (Figure 1A). The UC-MSCs undergoing osteogenic differentiation displayed bright red color after Alizarin-red staining (Figure 1B). The phenotype of UC-MSC showed positive expression of CD105 (95.9\%), CD73 (99.2\%), and CD90 $(99.9 \%)$, and negative expression of hematopoietic lineage marker $(2.0 \%)$ (Figure 1).

A

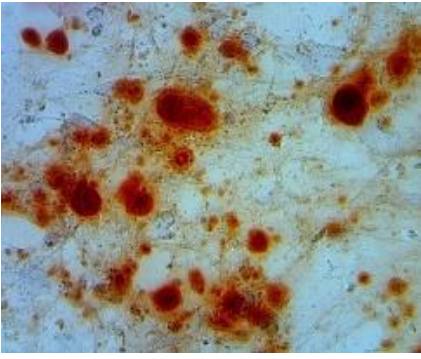

B

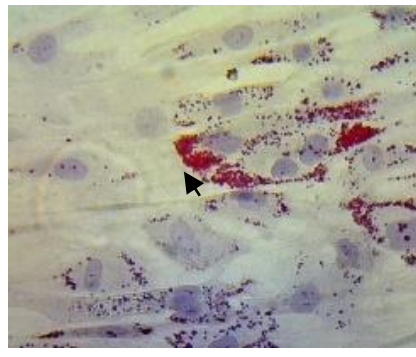

$\mathrm{C}$

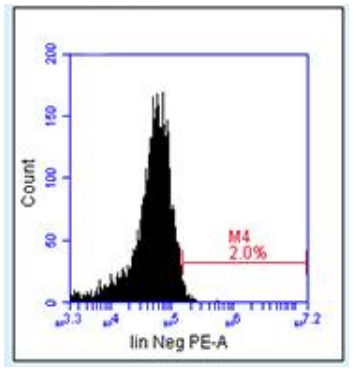

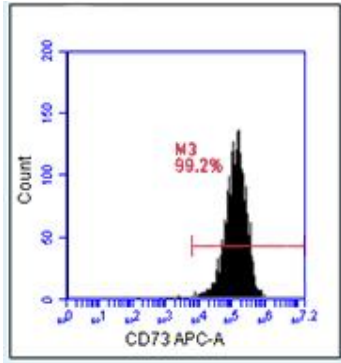

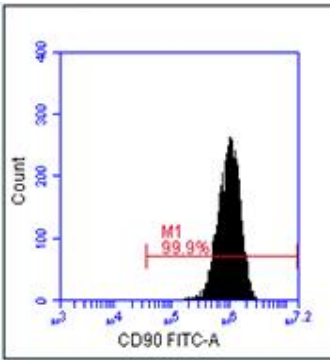

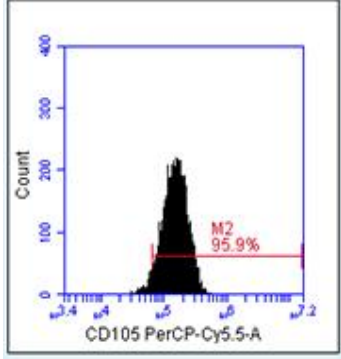

Figure 1. (A) Adipogenicity differentiation showed by the formation of lipid droplets and oily vesicles (Black arrow) after Oil Red O Staining, (B) calcium deposition after Alizarin-red staining (black arrow); (C) low expression of lin Neg and high expression of CD73, CD90, and CD105

The SGOT and SGPT analysis was performed to determine liver function capacity. This study shows that there was a significant decrease in SGOT concentration $(\mathrm{P}<0,05)$. P2 group treatment showed the lowest concentration of SGOT among the treatment (Figure B). In the same time, the administration of UC-MSCs reduce SGPT concentration insignificantly $(\mathrm{P}>0,05)$ (Figure 2C). In addition, both SGOT and SGPT concentration was linearly reduced along with the increase of UC-MSC dose.

\section{DISCUSSION}

Liver fibrosis results in the tremendous loss of hepatic cellular function, leading to liver failure and in prolonged time might cause death. The continuous fibrogenesis is caused by prolonged inflammation, thus controlling inflammation would be the key to reduce $\mathrm{LF}^{17}$. In addition, regenerating the damage hepatic cell would recover the liver function and provide great clinical value. Mesenchymal stem cells are a promising agent to reduce LF and recover the liver function since MSC has the immunomodulator properties and differentiation capacity into hepatic cell ${ }^{13,18}$. In this study we injected MSCs into LF rat models using intravenous routes in two doses to determine the optimum doze of MSC to recover liver function. 


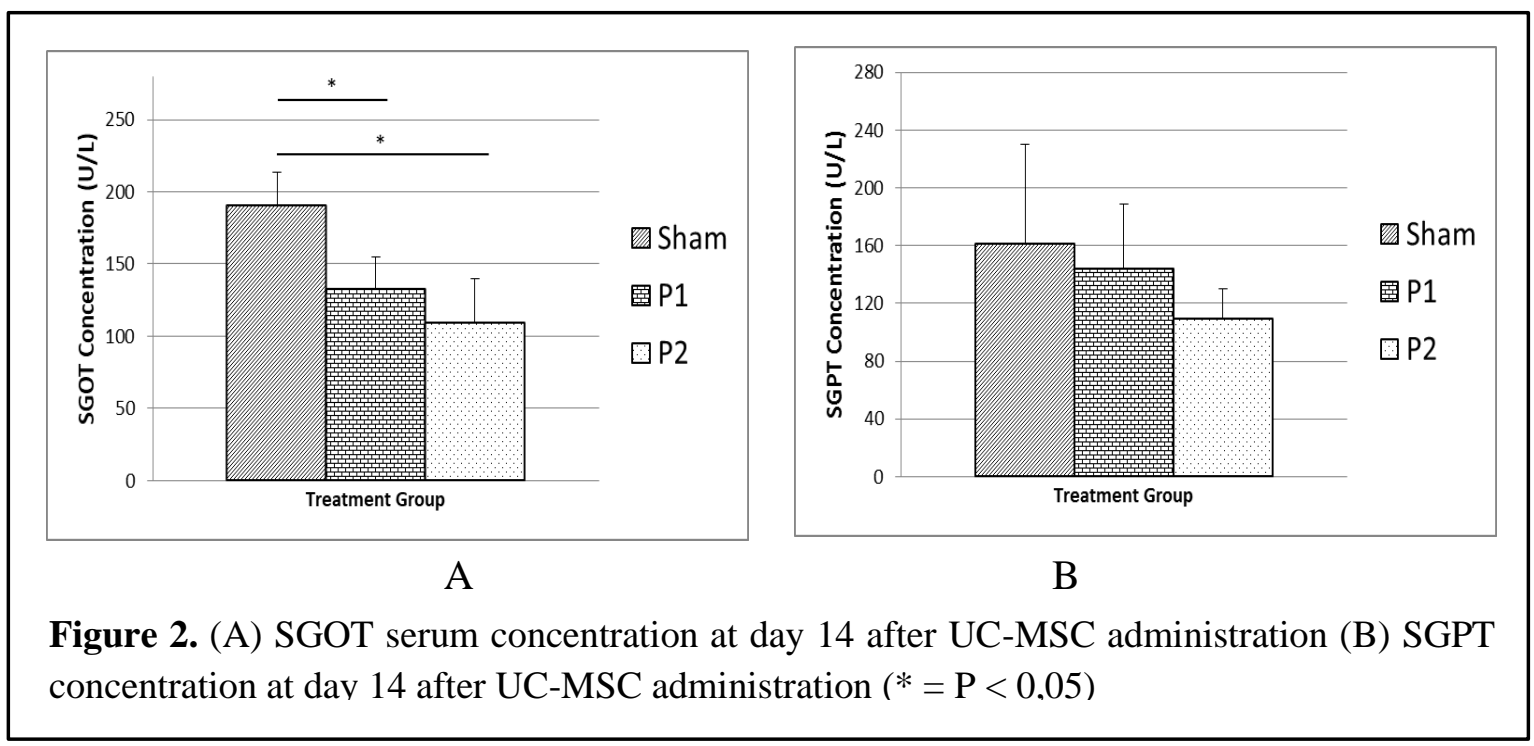

In this study we found that the concentration of SGPT and SGOT from both MSC treatment group significantly decreased compared with both sham groups. We also found that high dose of MSC reduce SGOT concentration significantly compared to low dose of MSC. Our findings suggested that high dose of MSCs more optimum to regenerate damage liver cell than low dose. Ther role of MSC in reducing SGPT and SGOT in LF rat model was related to their ability to control inflammation and acceleratee the transition of inflammation to the proliferation phase ${ }^{19,20}$. We hypothesize that high dose of MSCs increase the number of survived cell post administration to migrate to the injured area. In the injured area, MSCs inhibit the activation of macrophages resident, by releasing interleukin (IL)-10, IL-1 ra, prostaglandin E2 (PGE2), and transforming growth factor (TGF) $\beta^{21-23}$. The control of the inflammation process may result in the initiation of the regeneration phase and correlated with the acceleration of liver function repair that characterized by the significant decreases in SGPT and SGOT levels.

Damaged cells produce hypoxia-inducible factor (HIF)-1a that stimulate the release of several chemoattractant, including hepatocyte growth factor (HGF), SDF-1, and VEGF ${ }^{24}$. MSCs respond to these signals by expressing adhesion molecule, CD44, in addition to VLA-4, CXCR-4, and cMet. Particulary, in MSCs, CD44 activates the CD44-Src-integrin signaling axis and acts as VEGFR-213 co-receptor leading to MSCs homing initiation to the injured area. This mechanism trigger CD44-VEGF and VLA-4-VCAM-1 binding in capillary endothelial cells, facilitating the transmigration to injured areas ${ }^{25}$. However in this study the signalling procceses were not evaluated and become the study limitation.

\section{CONCLUSION}

The transplantation of MSCs ameliorated LF by reducing SGPT and SGOT concentration.

\section{ACKNOWLEDGEMENT}

We would like to thank SCCR Laboratory for supoorting this study

\section{CONFLICT OF INTEREST}

We declare that we have no conflict of interest. 


\section{AUTHORS' CONTRIBUTION}

ADA contribution in conceived and designed the analysis and wrote the paper. AP contribution in collected the data and contributed analysis tools. BAN contribution in performed analysis and make an animal model.

\section{FUNDING}

None

\section{AUTHOR DETAILS}

${ }^{1}$ Department of Postgraduate Biomedical Science, Medical Faculty, Sultan Agung Islamic University, Semarang, Indonesia

${ }^{2}$ Stem Cell and Cancer Research, Medical Faculty, Sultan Agung Islamic University, Semarang, Indonesia

${ }^{3}$ Medical Faculty, Sultan Agung Islamic University, Semarang, Indonesia

\section{REFERENCES}

1. Roehlen N, Crouchet E, Baumen TE. Liver Fibrosis : Mechanistic Concepts And.; 2020.

2. Shah B, Shah G. Antifibrotic effect of heparin on liver fibrosis model in rats. World J Gastrointest Pharmacol Ther. 2012;3(6):86.

3. Asrani SK, Devarbhavi H, Eaton J, Kamath PS. Burden of liver diseases in the world. J Hepatol. 2019;70(1):151-171.

4. Martin P, Dimartini A, Feng S, Brown R, Fallon M. Evaluation for liver transplantation in adults: 2013 practice guideline by the American Association for the Study of Liver Diseases and the American Society of Transplantation. Hepatology. 2014;59(3):1144-1165.

5. Fu X, Fang L, Li X, Cheng B, Sheng Z. Enhanced wound-healing quality with bone marrow mesenchymal stem cells autografting after skin injury. Wound Repair Regen. 2006;14(3):325-335.

6. Wu Y, Chen L, Scott PG, Tredget EE. Mesenchymal Stem Cells Enhance Wound Healing Through Differentiation and Angiogenesis. Stem Cells. 2007;25(10):2648-2659.

7. Nugraha A, Putra A. Tumor necrosis factor- $\alpha$-activated mesenchymal stem cells accelerate wound healing through vascular endothelial growth factor regulation in rats. Universa Med. 2018;37(2):135.

8. Galipeau J, Krampera M, Barrett J, et al. International Society for Cellular Therapy perspective on immune functional assays for mesenchymal stromal cells as potency release criterion for advanced phase clinical trials. Cytotherapy. 2015;18(2):151-159.

9. Kolios G, Moodley Y. Introduction to stem cells and regenerative medicine. Respiration. 2012;85(1):310.

10. Das M, Sundell IB, Koka PS. Adult Mesenchymal Stem Cells and their potency in the Cell-Based Therapy. J Stem Cells. 2013;8(1):1-16.

11. Putra A, Antari AD, Kustiyah AR, et al. Mesenchymal stem cells accelerate liver regeneration in acute liver failure animal model. Biomed Res Ther. 2018;5(11):2802-2810.

12. Hamra NF, Putra A, Tjipta A, Amalina ND, Nasihun T. Hypoxia mesenchymal stem cells accelerate wound closure improvement by controlling $\alpha$-smooth muscle actin expression in the full-thickness animal model. Open Access Maced J Med Sci. 2021;9:35-41.

13. Gazdic M, Arsenijevic A, Markovic BS, et al. Mesenchymal stem cell-dependent modulation of liver diseases. Int J Biol Sci. 2017;13(9):1109-1117.

14. Tsuchiya A, Takeuchi S, Watanabe T, et al. Mesenchymal stem cell therapies for liver cirrhosis: MSCs as "conducting cells" for improvement of liver fibrosis and regeneration. Inflamm Regen. 2019;39(1):49. 
15. Chen L, Tredget EE, Wu PYG, Wu Y, Wu Y. Paracrine factors of mesenchymal stem cells recruit macrophages and endothelial lineage cells and enhance wound healing. PLoS One. 2008;3(4).

16. Sengupta M, Sharma GD, Chakraborty B. Hepatoprotective and immunomodulatory properties of aqueous extract of Curcuma longa in carbon tetra chloride intoxicated Swiss albino mice. Asian Pac J Trop Biomed. 2011;1(3):193-199.

17. Ueha S, Shand FHW, Matsushima K. Cellular and molecular mechanisms of chronic inflammationassociated organ fibrosis. Front Immunol. 2012;3(APR):1-6.

18. Gao F, Chiu SM, Motan DAL, et al. Mesenchymal stem cells and immunomodulation: Current status and future prospects. Cell Death Dis. 2016;7(1).

19. Sargent A, Miller RH. MSC Therapeutics in Chronic Inflammation. Curr Stem Cell Reports. 2016;2(2):168-173.

20. Pers YM, Ruiz M, Noël D, Jorgensen C. Mesenchymal stem cells for the management of inflammation in osteoarthritis: State of the art and perspectives. Osteoarthr Cartil. 2015;23(11):2027-2035.

21. Madrigal M, Rao KS, Riordan NH. A review of therapeutic effects of mesenchymal stem cell secretions and induction of secretory modification by different culture methods. J Transl Med. 2014;12(1):1-14.

22. Putra A, Ridwan FB, Putridewi AI, et al. The role of tnf- $\alpha$ induced mscs on suppressive inflammation by increasing tgf- $\beta$ and il-10. Open Access Maced J Med Sci. 2018;6(10):1779-1783.

23. Liu F, Qiu H, Xue M, et al. MSC-secreted TGF- $\beta$ regulates lipopolysaccharide-stimulated macrophage M2-like polarization via the Akt/FoxO1 pathway. Stem Cell Res Ther. 2019;10:345.

24. Chen $\mathrm{L}, \mathrm{Xu} \mathrm{Y}$, Zhao J, et al. Conditioned medium from hypoxic bone marrow-derived mesenchymal stem cells enhances wound healing in mice. PLoS One. 2014;9(4).

25. Tumbarello DA, Turner CE. Hic-5 Contributes to Transformation Through a RhoA / ROCK-dependent Pathway. J Cell Physiol. 2006;211(3)(May):736-747. 